\title{
Zur Debatte über Verfassungsreformen in Chile
}

\author{
Von Peter Gailhofer, Zürich*
}

\section{A. Einleitung}

Im Gegensatz zu anderen südamerikanischen Staaten, die nach dem Ende der dort in der zweiten Hälfte des 20. Jahrhunderts etablierten Militärdiktaturen entweder zu ihren vorautoritären Verfassungen „,zurückkehrten“ oder neue Verfassungstexte verabschiedeten, konnte die demokratische Opposition in Chile zum Ende der Herrschaft Augusto Pinochets im Jahr 1990 nur die Modifikation und teilweise Demokratisierung der unter der Militärdiktatur verabschiedeten Verfassung durchsetzen. ${ }^{1}$ Das in der Politischen Verfassung von $1980^{2}$ fixierte ,institutionelle Erbe“ des Militärregimes bedingte in den folgenden Jahren die Möglichkeiten politischer Reformen, ${ }^{3}$ die Verfassung hatte so einen entscheidenden Einfluss auf den Verlauf des Übergangs Chiles zur Demokratie.

Wenige Jahre nach einer umfangreichen Verfassungsreform im Jahr 2005 wird in Chile weiterhin intensiv über neuerliche Reformen diskutiert. Wie bisher bestimmt dabei die Verfassung und die mit ihr noch immer teilweise konservierte institutionelle Ordnung Pinochets ${ }^{5}$ einerseits das Verfahren und die politische Machbarkeit von Reformen und steht andererseits angesichts ihres illegitimen Ursprungs in ihrer Gesamtheit als Gegenstand von Reformbestrebungen im Zentrum der Debatte. Dieser Beitrag soll nach einer kurzen Darstellung der historischen und institutionellen Hintergründe einige der wesentlichen Inhalte und Argumente der Reformdebatte aufzeigen.

* Peter Gailhofer, dipl. jur., Wissenschaftlicher Assistent am Lehrstuhl für Rechtstheorie, Rechtssoziologie und Internationales Öffentliches Recht, Universität Zürich.

E-mail: peter.gailhofer@rwi.uzh.ch

1 Vgl. Ellen Bos, Verfassungsgebung und Systemwechsel, Wiesbaden 2004, S. 120.

2 Constitución Política de la República de Chile, abrufbar unter: http://www.leychile.cl/Navegar? idNorma $=242302$.

3 Kamel Cazor Aliste, Democrácia y Constitución en Chile, Revista de Derecho (Valvidia), 11 (2000), S. 31.

4 Vgl. Fredrik Uggla, Chiles Constitutional Changes, Latin American Politics \& Society 47.2 (2005), S. 70.

5 Vgl. Pablo Ruiz Tagle, in: Simposio: Reformas del ano 2005 a la Constitución de la Republica de Chile, Anuario de Derechos Humanos 2006, S. 80 f. 


\section{B. Hintergründe}

\section{Die Herkunft der Verfassung Chiles und das Ende der Diktatur}

Chiles Verfassung wurde zwischen 1978 und 1980 unter der Militärdiktatur Augusto Pinochets erarbeitet, durch ein - allerdings angesichts der formalen und faktischen Umstände seines Zustandekommens überaus fragwürdiges ${ }^{6}$ - Plebiszit angenommen und trat am 11.3.1981 in Kraft. Der vielfach kritisierte autoritäre und antidemokratische Charakter dieser Verfassung zeigte sich insbesondere in der starken Stellung des Präsidenten gegenüber dem Parlament, dessen weit reichenden Befugnissen im Ausnahmezustand, dem fehlenden Grundrechtsschutz im Ausnahmezustand, dem parlamentarischen Einfluss ernannter Senatoren (Eliteprinzip), pluralismusfeindlicher Mechanismen und der übermächtigen Stellung des Militärs im Staatsgefüge. ${ }^{7}$ Nachdem der Versuch der Militärjunta, Pinochet nach Ablauf seiner Amtszeit im Jahr 1988 für weitere acht Jahre im Präsidentenamt zu etablieren, aufgrund des ablehnenden Ausgangs des hierüber entscheidenden Plebiszits gescheitert war, ${ }^{8}$ konnte nach und nach ein Demokratisierungsprozess in Gang kommen. ${ }^{9}$ Die in der Folge dieser Niederlage Pinochets stattfindenden Verhandlungen zwischen der Junta und der Concertación, dem Bündnis der demokratischen Opposition, über eine Reform der Verfassung, können als eines der maßgeblichen Ereignisse für den weiteren Übergang Chiles zur Demokratie betrachtet werden. Zum einen brachte die aus diesen Vereinbarungen hervorgegangene erste Verfassungsreform im Jahr 1989 insgesamt 54 Änderungen des Verfassungstextes mit sich, die insbesondere auf die Ermöglichung eines politischen Pluralismus, die Erhöhung der Anzahl der zivilen Mitglieder des einflussreichen nationalen Sicherheitsrats, eine verbesserte Integration internationaler Verträge ins nationale Recht und diverse Beschränkungen der übermächtigen Exekutive abzielten. So konnte der für ein „Aufkeimen“ der Demokratie ${ }^{10}$ erforderliche minimale konstitutionelle Freiraum geschaffen werden, auch wenn die demokratischen und rechtsstaatlichen Defizite

Vgl. J. Esteban Montes/ Tomás Vial, The role of constitution building processes in democratization, Case Study Chile, http://www.idea.int/cbp/upload/CBP-Chile.pdf, S. 5.

Manfred Braatz, Demokratie in Chile?, Jahrbuch des Öffentlichen Rechts 41 (1993), S. 498.

Die Durchführung eines Plebiszits über die Fortführung Pinochets Präsidentschaft war in der Verfassung selbst vorgesehen. Bereits seit Mitte der achtziger Jahre wurde in der demokratischen Opposition darüber diskutiert, diese Regelung zu nutzen, um Pinochet ,an der Urne“ zu schlagen, Uggla, Fn. 4, S. 51, 55 f.

Ausführlich zur Verlauf des Transitionsprozesses: Julia Paley, Marketing democracy: power and social movements in post-dictatorship Chile, Berkeley/Los Angeles/London, 2001, S. 92 ff.; S.a. Braatz, Fn. 7, S. 499 ff.

10

José Manuel Martínez Sierra/ Covadonga Ferrer, La Reforma Constitucional en Chile, Paper zur Veranstaltung „Jornada sobre orientacion y metodo del Derecho Constitucional“, Madrid, 16.11. 2007, abrufbar unter http://www.uned.es/dpto-derecho-político/Comunicación Martinez Ferrer. pdf, S. 2. 
der Verfassung in erheblichem Umfang fortbestanden. ${ }^{11}$ Zum anderen stellten die Verhandlungen den ersten Fall von politischen Vereinbarungen zwischen der Concertación und Pinochets Unterstützern aus den Reihen der rechtsgerichteten Parteien, der Streitkräfte und der wirtschaftlichen Elite dar, einer Konsenspolitik, ${ }^{12}$ welche Chiles Demokratisierung auch während der folgenden Jahre charakterisieren sollte. ${ }^{13}$ Ein folgenreiches Ergebnis dieses ersten Konsenses zwischen den antagonistischen politischen Kräften war die - trotz aller sonstigen Änderungen - weitgehende Beibehaltung der in Pinochets Verfassung verankerten Staatsorganisation und insbesondere derjenigen Regelungen, welche die Notwendigkeit eines sehr breiten politischen Konsenses bei wichtigen, insbesondere verfassungsrechtlichen Reformprojekten der folgenden Jahre festschreiben sollten. Diese institutionellen Regelungen - wie etwa der „kontramajoritäre“ Einfluss des Militärs oder Chiles binominales Wahlsystem $-{ }^{14}$ waren von den Verfassern der „Constitución Política“ zu dem Zweck entworfen worden, die reformerischen Spielräume demokratischer Mehrheiten zu beschränken und den Einfluss von der Junta nahestehenden Kreisen zu sichern. ${ }^{15}$

Angesichts der wegen Pinochets fortbestehender Staatsorganisation perpetuierten Einflussmöglichkeiten der ehemals der Diktatur verpflichteten politischen und gesellschaftlichen Kreise und Institutionen war daher auch im Zuge der 16 folgenden Verfassungsreformen zwischen 1991 und $2003^{16}$ nur die schrittweise Eliminierung der ,,autoritären Enklaven “ ${ }^{17}$ in der Verfassung möglich. Auch diese konnten die autokratischen Grundzüge der Staatsordnung nicht beseitigen. ${ }^{18}$ Während der Amtszeiten der Präsidenten Patricio Aylwin und Eduardo Frei Ruiz Tagle konnten so etwa erste Reformen der kommunalen und regionalen Verwaltung, eine Reduktion der Amtszeit des Präsidenten von acht auf sechs Jahre und eine umfangreiche Justizreform durchgesetzt werden. ${ }^{19}$ Am Fortbestehen einer Vielzahl militaristischer und demokratisch äußerst fragwürdiger Regelungen zeigte sich jedoch

Braatz, Fn. 7, S. 499 ff. mit ausführlicher Argumentation.

Vgl. Fazio, El Ciudadano v. 15.2.2010.

Vgl. Uggla, Fn. 5, S. 51. Cazor Aliste, Fn. 3, S. 31.

S.u. C.I.

Vgl. Portales, El Clarin, 17.3.2010 m.w.N., Cazor Aliste Fn. 3, S. 31.

Eine aktualisierte Auflistung aller Verfassungsreformen seit 1989 ist unter http://www.bcn.cl/lc/ cpolitica/leyes-reforma-constitucion einzusehen.

So die in der chilenischen Staatswissenschaft übliche Bezeichnung (,enclaves autoritarios“) für die institutionellen Hinterlassenschaften des Militärregimes, Martínez Sierra/ Ferrer, Fn. 10, S. 2 , vgl. auch Francisco Cumplido Cereceda, Reforma Constitucional en Chile, Anuario de Derecho Constitucional Latinoamericano 2006, S. 105.

Cazor Aliste, Fn. 3, S. 31. Vgl. auch Pablo Ruiz-Tagle, El segundo piso de La Moneda el pato volador, el pato cojo y los desafíos de la constitución gatopardo, http://www.pabloruiz-tagle.cl/ blog/publicaciones-formato-electronico/, S. 4.

Cumplido Cereceda, Fn. 17, S. 106. 
auch zum Zeitpunkt des Amtsantritts von Präsident Ricardo Lagos im März 2000 noch der „verlängerte Schatten Pinochets“. 20

\section{Die Verfassungsreform des Jahres 2005}

Mit dem Anspruch, die ,,autoritären Enklaven“ aus der Verfassung zu tilgen und so Chiles Demokratisierungsprozess zu vollenden, ${ }^{21}$ wurden im August 2005, nach einem bereits fünf Jahre zuvor mittels zweier Initiativen aus dem Senat ${ }^{22}$ in die Wege geleiteten Reformprozesses weitere 58 Verfassungsänderungen verabschiedet. ${ }^{23}$ Erarbeitet wurde die Reform von einer zwischen den politischen Kräften vermittelnden, paritätisch besetzten Kommission; mehrere wesentliche Verfassungsänderungen kamen infolge der Ausübung des präsidentiellen Vetorechts zustande. ${ }^{24}$ Einige der auffälligsten institutionellen Hinterlassenschaften des Militärregimes wurden mit der Verabschiedung dieser Reform durch eine breite parlamentarische Mehrheit beseitigt; insbesondere konnten der politische Einfluss und die Präsenz des Militärs in den Verfassungsorganen eliminiert werden. So ist die Stellung der Teilstreitkräfte und der Polizei als eigenständige Staatsgewalt, als exklusive „Hüter der Verfassung“, ${ }^{25}$ nach dem reformierten Verfassungstext nicht mehr vorgesehen. Zudem entfällt das institutionelle Gewicht des zu einem erheblichen Anteil mit Vertretern der Streit- und sonstigen Sicherheitskräfte besetzten ${ }^{26}$ nationalen Sicherheitsrats: Dessen weitgehende Kontrollbefugnisse und Mitspracherechte gegenüber sämtlichen Staatsorganen und sein erheblicher Einfluss auf die personelle Zusammensetzung von Senat und Verfassungsgericht wurden abgeschafft. ${ }^{27}$ Der Sicherheitsrat fungiert nunmehr lediglich als Bera-

20

21

Martinez Sierra/ Ferrer, Fn. 10, S. 17.

Präsident Ricardo Lagos hatte bereits zu Beginn seiner Präsidentschaft im Jahr 2000 eine Verfassung versprochen, ,die den Test einer vollen Demokratie besteht“, zitiert nach The Economist v. 23.10.2004, S. 36.

Aufgrund der voneinander unabhängigen Gesetzesinitiativen von Senatoren der ,Allianza por Chile“ ( $N^{\circ}$ Boletín: 2526-07) und der Concertación por la Democracia ( $N^{\circ}$ Boletín: 2534-07), abrufbar unter http://sil.senado.cl/pags/index.html.

Ley $\mathrm{N}^{\circ} 20.050$ vom 26.8.2005, abrufbar unter http://www.bcn.cl/leyes/pdf/original/241331.pdf. Konsolidiert mit dem Decreto Supremo $\mathrm{N}^{\circ}$ 100, vom 22.9.2005, abrufbar unter http://www. bcn.c1/leyes/pdf/actualizado/242302.pdf.

Präsident Lagos konnte so 27 Verfassungsänderungen durchsetzen, vgl. die Darstellung des Reformprozesses bei Martinez Sierra/ Ferrer, Fn. 10, S. 2 ff.

Vgl. Braatz, Fn. 7, S. 525.

Bestehend aus dem Präsidenten der Republik, dem Präsidenten des Senats und dem Präsidenten des Obersten Gerichtshofs, dem ,,contralor general“, jeweils dem Kommandanten der Armee, der Seestreitkräfte und der Luftwaffe, sowie dem Generaldirektor der Polizeikräfte

Der Sicherheitsrat hatte bis dahin die Kompetenz, vier der insgesamt neun ,institutionellen“ Senatoren sowie zwei der sieben Mitglieder des Verfassungsgerichts zu ernennen, Cumplido Cereceda, Fn. 17, S. 107. 
tungsorgan in Sicherheitsfragen. ${ }^{28}$ Auch das durch ernannte Senatoren ${ }^{29}$ und Senatoren auf Lebenszeit $^{30}$ verkörperte, die demokratische Repräsentation innerhalb der Legislative erheblich verzerrende sogenannte Eliteprinzip ist mit der Reform Geschichte. Sämtliche 38 Sitze in Chiles Senat sind seit 2006, als die Amtszeit der letzten jener ,institutionellen Senatoren“ endete, durch öffentliche Wahlen besetzt. Mit dem Ende der ,autoritären Enklaven“ und einiger weiterer ${ }^{31}$ Modifikationen der chilenischen Verfassung ist die Bedeutung der Reform des Jahres 2005 für den Demokratisierungsprozess Chiles grundsätzlich unbestritten. ${ }^{32}$ Über die Notwendigkeit weiterer Reformen wird jedoch weiterhin kontrovers diskutiert.

\section{Zur aktuellen Diskussion}

Wenige Jahre nach der letzten großen Verfassungsreform stehen in Chile weiterhin eine Vielzahl von Forderungen nach weiteren Verfassungsreformen auf der politischen Agenda; auch der neue, zum ersten Mal seit dem Ende der Militärdiktatur aus dem Lager der Rechten stammende Präsident fordert Reformen zur „Verbesserung der Demokratie“. ${ }^{33}$ Die Ursachen für die aktuelle Verfassungsdebatte sind vielfältig: Zivilgesellschaftliche Bewegungen und nationale Minderheiten drängen auf Änderungen des Verfassungsrechts im Sinne ihrer politischen Forderungen, seit der letzten Reform aufgeschobene Verfassungsmodifikationen warten weiterhin auf ihre Realisierung, die verfassungsmäßige Stellung und Organisation der Verfassungsorgane, wie dem Präsidenten oder dem Verfassungsgerichtshof, wird als reformbedürftig erachtet und eine intensive akademische und politische Diskussion stellt die Legitimität der Verfassung aufgrund ihrer Herkunft und ideologischen

Zu den Veränderungen bzgl. der verfassungsmässigen Position der Streit- und Sicherheitskräfte durch die Reform 2005 siehe die umfangreiche Darstellung bei Martinez Sierra/ Ferrer, Fn. 10, S. $10 \mathrm{ff}$.

Zwei der Senatoren wurden vom Präsidenten, drei vom Obersten Gerichtshof und vier (aus der Führungsriege der Streit- und Polizeikräfte zu besetzende Kandidaten) vom Sicherheitsrat ernannt. Diesen neun nicht demokratisch legitimierten Senatsmitgliedern standen, nach einer Erhöhung des Anteils der gewählten Senatoren durch die Reform von 1989, 38 vom Volk gewählte Senatsmitglieder gegenüber, vgl. Cumplido Cereceda, Fn. 17, S. 109.

Ein Senatssitz auf Lebenszeit wurde den ehemaligen Präsidenten zugestanden.

Zu nennen sind beispielsweise Beschränkungen für die Verhängung des Ausnahmezustands, die Ergänzung des zur Regelung der Staatsangehörigkeit bislang geltenden ius soli dergestalt, dass nunmehr auch im Ausland geborene Kinder chilenischer Eltern die Staatsangehörigkeit erwerben, oder die Aufnahme der Verpflichtung zur Solidarität zwischen den Regionen in die Verfassung, vgl. die Übersicht der Bibliothek des chilenischen Nationalkongresses unter http://www.bcn.cl/ carpeta_temas/temas_portada.2005-10-24.0525136469.

Vgl. die Beiträge von Jose Luis Cea/ Rodrigo Correa/ Enrique Navarro u.a. in: Simposio: Reformas del ano 2005 a la Constitución, Fn. 5, S. $101 \mathrm{ff}$.

33 Vgl. etwa http://www.chilesomostodos.gov.cl/index.php?option=com_content\&task=view\&id= $261 \&$ Itemid $=36$. 
Grundausrichtung grundsätzlich in Frage. Einige Themen dieser Verfassungsdebatte sollen im Folgenden kurz vorgestellt werden.

\section{Die aufgeschobene Reform des Wahlsystems und die „organischen Verfassungsgesetze“}

Einige der auffälligsten fortbestehenden institutionellen Hinterlassenschaften der Militärjunta Pinochets betreffen Regelungen, welche die politischen Spielräume für bedeutsame Reformen zugunsten der - bis zu der Präsidentschaftswahl dieses Jahres chronisch minoritären - politischen Rechten einzuschränken bestimmt waren, indem gesellschaftliche Mehrheitsverhältnisse durch eine eigentümliche Variante parlamentarischer Repräsentation neutralisiert und zugleich hohe Quoren für die Regelung bestimmter Rechtsbereiche in der Verfassung verankert wurden. Insbesondere mit dem sogenannten ,binominalen“ System besteht eine unter dem Gesichtspunkt der demokratischen Repräsentativität der Legislative äußerst fragwürdige und zugleich hinsichtlich der Machbarkeit neuerlicher Reformen entscheidende Regelung trotz sämtlicher Reformbestrebungen fort. Dieses Wahlsystem ,sui generis “ ${ }^{34}$ bestimmt, dass - auf der Basis von durch die Parteien bzw. Parteienbündnisse aufzustellenden Listen - für jede der beiden Kammern des Nationalkongresses jeweils zwei Abgeordnete bzw. Senatoren je Wahlkreis gewählt werden. Die jeweiligen Parteilisten dürfen maximal zwei Kandidaten enthalten; um beide Kandidaten einer Partei oder eines Parteienbündnisses ins Amt zu bringen, muss deren Liste mindestens doppelt so viele Stimmen erhalten wie diejenige der zweitstärksten Parteienliste. Dies führt dazu, dass das erfolgreichste Oppositionsparteienbündnis bereits mit der Hälfte der Stimmen ebenso viele Sitze im Kongress besetzen kann wie die Wahlsieger. Kleinere Parteien bleiben dagegen, selbst wenn ein relevanter Anteil der Wählerstimmen auf sie entfällt, gänzlich unberücksichtigt und damit von der Repräsentation in Abgeordnetenhaus und Senat ausgeschlossen; ethnische und regionale Minderheiten und andere marginalisierte Bevölkerungsgruppen sind in der chilenischen Legislative daher grundsätzlich unterrepräsentiert. ${ }^{35}$ Das stark asymmetrische binominale Wahlsystem resultiert so in einer chronischen „Patt-Situation“ ${ }^{36}$ zwischen den beiden großen nationalen Parteienbündnissen, der Mitte-Links-Koalition der Concertación und der ursprünglich der Diktatur verpflichteten „Allianza por Chile“ in der

Dies, da sich dieses weder als Mehrheits- noch als Verhältniswahl einordnen lässt, vgl. Pablo Ruiz Tagle, Kommentar zum Vortrag von Enrique Silva Cimma, Seminar „Chile debe tener una nueva Constitución“" an der Universidad Central in Santiago de Chile vom 25. 8. 2008, abrufbar unter: http://www.pabloruiz-tagle.cl/blog/2008/10/08/chile-debe-tener-una-nueva-Constitución-seminario-universidad-central-25-de-agosto-2008/.

$\mathrm{Zu}$ den Auswirkungen des binominalen Systems ausführlich die Veröffentlichung von FLACSO Chile (Facultad Latinoamericana de Ciencias Sociales), Efectos del Sistema Binominal, http:// issuu.com/flacso.chile/docs/e_binominal. S.a. Braatz, Fn. 7, S. 509; Cumplido Cereceda, Fn. 17, S. 109. 
chilenischen Legislative, so dass für gesetzgeberische Reformen grundsätzlich ein breiter parlamentarischer Konsens notwendig ist. Ergänzt wird dieser „,bewahrende“ wahlrechtliche Mechanismus durch die Institution „organischer Verfassungsgesetze“ („leyes orgánicas constitucionales“) die eine erhebliche Anzahl im Einzelnen in der Verfassung definierter, politisch bedeutsamer Rechtsbereiche zu regeln haben. Die Verabschiedung, Änderung oder Abschaffung eines „,organischen Verfassungsgesetzes“ bedarf einer Mehrheit von 4/7 in beiden Kammern des Nationalkongresses, ${ }^{37}$ so dass eine Reihe verbliebener institutioneller Hinterlassenschaften Pinochets einen erhöhten verfassungsrechtlichen Bestandschutz genießen. $^{38}$

Im Zuge der Reform des Jahres 2005 kam eine Änderung des Wahlsystems nicht zustande, vielmehr wurde auf die Bestimmung des Wahlsystems im Verfassungstext verzichtet und diese auf den einfachen Gesetzgeber übertragen. Da auch die für diese Wahlrechtsreform mittels eines „organischen Verfassungsgesetzes“ erforderlichen Mehrheiten angesichts der - aufgrund des Wahlsystems zustande gekommenen - parlamentarischen Mehrheitsverhältnisse nicht zu erreichen sind, konnte diese Reform bis heute nicht realisiert werden. Die verbleibenden ,autoritären Enklaven“ des binominalen Wahlsystems und der „organischen Verfassungsgesetze“ bleiben daher aufgrund der aus ihnen resultierenden demographischen Verzerrungen der demokratischen Repräsentation und der institutionellen Beschränkung der Möglichkeiten und Reichweite neuerlicher Verfassungsreformen Gegenstand nationaler wie internationaler Kritik $^{39}$ und stehen allem Anschein nach auf der politischen Agenda auch des neuen Präsidenten Piñera. ${ }^{40}$ Ob zukünftige Reformbestrebungen die erforderliche Mitwirkung derjenigen parlamentarischen Kreise mobilisieren können, die durch eine Änderung der entsprechenden Regelungen ihren privilegierten Einfluss verlieren würden, bleibt abzuwarten. ${ }^{41}$

\section{Die Stellung des Präsidenten}

Aufgrund der in wesentlichen Zügen fortbestehenden Kompetenzverteilung nach der Verfassung von 1980 ist Chiles Regierungssystem weiterhin von einer überaus starken Stellung des Präsidenten geprägt. Dieser ,gesteigerte Präsidentialismus “42 ist, neben umfassenden

Art. 66 Abs. 2 der Politischen Verfassung Chiles.

Vgl. Lukas Oberndorfer, Transnationalisierung des Rechts, Transitional Constitutionalism und Internationales Verfassungsrecht, ICL-WorkingPaper 01/2007 (www.icl-workshop.com).

Vgl. etwa den Bericht des Ausschusses für Menschenrechte des IPbpR vom 17.4.2007, S. 5.

Siehe http://www.chilesomostodos.gov.cl/index.php?option=com_content\&task=view\&id=261\& Itemid=36. S.a. http://www.lanacion.cl/pinera-al-pizarron-por-reformas-politicas/noticias/201002-16/221448.html.

Den Reformbestrebungen der letzten Regierung stand die Ablehnung der Abgeordneten der Alianza por Chile weiterhin entgegen, siehe http://www.lanacion.cl/prontus_noticias_v2/site/ artic/20090519/pags/2009051 9173704.html

Vgl. Cumplido Cereceda, Fn. 17, S. 110. 
exekutiven Kompetenzen, insbesondere durch weit reichende Legislativfunktionen, Haushaltskompetenzen und erheblichen Einfluss auf die Besetzung der Gerichte gekennzeichnet. ${ }^{43}$ Im Zuge der Reform des Jahres 2005 konnte diese Machtkonzentration auf Seiten des Präsidenten der Republik zwar abgemildert werden: So wurden etwa die Amtszeit des Präsidenten von sechs auf vier Jahre verkürzt ${ }^{44}$ und damit an die Legislaturperiode des Parlaments angepasst, die präsidialen Legislativkompetenzen eingeschränkt und die, allerdings sanktionslose, ${ }^{45}$ Möglichkeit parlamentarischer Kontrolle der Regierung - mittels Interpellationsrecht und der Kompetenz zur Einrichtung von Untersuchungskommissionen durch das Repräsentantenhaus - eingeführt. ${ }^{46}$ Gleichwohl ist das weiterhin bestehende institutionelle Kräfteungleichgewicht zugunsten des Präsidenten, welches insbesondere auch angesichts der aus dem Wahlsystem ${ }^{47}$ resultierenden Unbeweglichkeit des Kongresses und der daraus folgenden relativen Bedeutungslosigkeit der Parteien ${ }^{48}$ festgestellt wird, Gegenstand reger Kritik. ${ }^{49}$ Die Befürworter einer neuerlichen Reform fordern dementsprechend eine weitere Beschränkung der Kompetenzen des Präsidenten und die Modifikation des Regierungssystems hin zu einer parlamentarischen oder „semipräsidentialen“ Demokratie. $^{50}$

\section{Die Debatte um Chiles Verfassungsgerichtshof}

Auch die chilenische Verfassungsgerichtsbarkeit wurde im Rahmen der Reformen des Jahres 2005 umfassend modifiziert. Insbesondere wurde der Modus der Besetzung des Gerichts insofern demokratisiert, als das in der Verfassung von 1980 vorgesehene Recht des Nationalen Sicherheitsrats zur Benennung zweier der ursprünglich sieben Verfassungs-

43

44

45

46

47

48

49

50

Vgl. zur Stellung des Präsidenten im Verfassungsgefüge Martinez Sierra/ Ferrer, Fn. 10, S. 6 ff.

Eine Wiederwahl des Präsidenten bleibt für die auf seine Amtszeit folgende Legislaturperiode ausgeschlossen.

Martinez Sierra/ Ferrer, Fn. 10, S. 9.

Vgl. Cumplido Cereceda, Fn. 17, S. 110 f.

S.o. C. I.

Cumplido Cereceda meint, dass die Parteien aufgrund der relativen Bedeutungslosigkeit des Kongresses ihre wichtigste Funktion in der Mobilisierung von Stimmen für die Wahl des Präsidenten haben, gleichwohl wegen ihrer fehlenden Regierungsverantwortung selbst für die ursprünglich von ihnen unterstützten Präsidenten zu politischen Hindernissen werden können, vgl. ders. Fn. 17, S. 111.

Martinez Sierra/ Ferrer kritisieren die Reformen insbesondere aufgrund der Sanktionslosigkeit der parlamentarischen Kontrollrechte als „lauwarm“ und unzureichend, vgl. dies. Fn. 10, S. 10; Francisco Zúñiga Urbina spricht angesichts der durch das Wahlsystem institutionalisierten „PattSituation“ im Parlament von einer „Verkrustung“ des politischen Systems, das zu einem „HyperPräsidentialismus“ führe, vgl. ders., Vieja - Nueva Constitución, Estudios Constitucionales (Universidad de Talca) 2007, S. 367 f.

Vgl. Zúñiga Urbina, Fn. 49, S. 363. 
richter nunmehr entfällt. ${ }^{51}$ Durch die Einführung einer Reihe zusätzlicher Verfahren der Verfassungskontrolle ${ }^{52}$ hat sich mit der Reform zudem das politische Gewicht des Verfassungsgerichts erheblich erhöht. ${ }^{53}$ Kritiker befürchten nun allerdings aufgrund der hierdurch entstandenen erheblichen Einflussmöglichkeiten einen demokratisch nicht legitimierten, „kontra-majoritären“ politischen Aktivismus des Gerichts und fordern Reformen zur Gewährleistung seiner demokratischen Kontrolle und rechtlichen Verantwortlichkeit, um so einer befürchteten Richteroligarchie und „Tyrannei der Werte“ entgegen zu treten. ${ }^{54}$ Der Opposition gegen eine „Diktatur der Weisen“" ${ }^{45}$ haben sich nach einem im April 2008 erfolgten Verbot des Verfassungsgerichts der Abgabe der „Pille danach“ durch staatliche Gesundheitszentren, auch etliche zivilgesellschaftliche Organisationen angeschlossen. 56

\section{Dezentralisierung}

Eine weitere Forderung der Befürworter neuerlicher Reformen betrifft einen ,profunden Wechsel der Regierung, Verwaltung und Entwicklung Chiles“ mittels einer Regionalisierung des Staatssystems. ${ }^{57}$ Eine Verbesserung der bürgerlichen Partizipationsmöglichkeiten auf regionaler Ebene, die Ausweitung der politischen Kompetenzen und bessere Versorgung der regionalen und lokalen Institutionen mit ökonomischen Ressourcen und eine verbesserte regionale Ausrichtung insbesondere der nationalen Wirtschaftspolitik sollen die effektive politische und ökonomische Dezentralisierung des Landes sicherstellen.

Ein im Oktober 2009 in Kraft getretenes „organisches Verfassungsgesetz“ zur Reform der regionalen Regierung und Verwaltung realisiert nur einen Teil dieser Forderungen, ${ }^{58}$

Nunmehr werden drei der zehn Verfassungsrichter vom Präsidenten der Republik ernannt, drei vom Obersten Gerichtshof, sowie je zwei von Senat und Abgeordnetenhaus gewählt, vgl. Artikel 92 der Politischen Verfassung der Republik Chile.

Unter anderem wurden Verfahren abstrakter, präventiver, fakultativer oder obligatorischer Normenkontrolle, sowie ein Organstreitverfahren eingeführt. Darüber hinaus besteht nunmehr die Kompetenz zur „,konkret-repressiven“, fakultativen Kontrolle der Verfassungsmäßigkeit von Gesetzen, die für ,unanwendbar“ oder verfassungswidrig erklärt werden können, siehe Art. 93 der Politischen Verfassung der Republik Chile; Cumplido Cereceda, Fn. 17, S. 108.

Cumplido Cereceda spricht vom Verfassungsgericht als einer „,neuen politischen Supermacht“ im chilenischen Staatsgefüge, Fn. 17, S. 108.

Vgl. Zúñiga Urbina, Fn. 49, S. 357, 368.

55 $\mathrm{Vgl}$. http://www.lanacion.cl/prontus_noticias_v2/site/artic/20080412/pags/20080412203730.html.

Vgl. http://www.lanacion.com.ar/nota.asp?nota_id=1006783, http://www.rel-uita.org/mujer/ mujeres_no_pue den_decidir.htm.

57 Vgl. Zúñiga Urbina, Fn. 49, S. 363.

58 Siehe http://www.subdere.gov.cl/1510/article-78618.html. 
und auch der neue Präsident Piñera sieht weiteren Handlungsbedarf ${ }^{59}$ - auch in diesem Bereich sind die Reformbestrebungen also noch nicht zu einem Abschluss gelangt.

\section{Bildungssystem}

In der „Revolution der Pinguine“ mobilisierten Schüler- und Studentenorganisationen im Frühjahr 2006 hunderttausende vor allem junger Chilenen zu Streiks in Schulen und Universitäten und groß angelegten Demonstrationen gegen das Bildungssystem. ${ }^{60}$ Im Zentrum der Proteste stand, neben allgemeinen Forderungen nach der Verbesserung der Qualität der Bildung, das LOCE („Ley Orgánica Constitucional de Enseñanza“), das „organische“61 Bildungsgesetz, welches aufgrund seiner neoliberalen Ausrichtung als Ursache der Defizienz des Bildungssystems betrachtet wurde. Kernelemente des LOCE, kurz vor dem Ende der Diktatur Pinochets im Jahr 1990 verabschiedet, waren die teilweise Privatisierung des Schulsystems, die Kommunalisierung des Bildungsauftrags und ein Subventionssystem für die Schulen; die Gewährleistung der Qualität des Bildungssystems wurde dem freien Wettbewerb überlassen. Der Staat als potentieller Garant des Grundrechts auf Bildung ${ }^{62}$ blieb dagegen marginalisiert. ${ }^{63}$

Nachdem ein durch die vormalige Präsidentin Michelle Bachelet eingerichtetes Beratergremium bereits im Jahr 2006 unter anderem die Ersetzung des LOCE empfohlen hatte, ${ }^{64}$ konnte nach langwierigem Ringen um die zum Erlass eines neuen „organischen Verfassungsgesetzes“ erforderliche Mehrheit im Parlament und zahlreichen Änderungen an dem ursprünglichen Reformvorschlag ${ }^{65}$ im August 2009 das „Ley General de Educación“ (LGE) erlassen werden. ${ }^{66}$ Die Änderungen gegenüber der bisherigen Rechtslage betreffen, neben einigen strukturellen Anpassungen, Regelungen zur Verbesserung der Transparenz des staatlichen Subventionssystems, sowie die Installation staatlicher Aufsichtsbehörden, wel-

Siehe http://www.chilesomostodos.gov.cl/index.php?option=com_content\&task=view\&id=61\& Itemid=36.

Siehe http://www.pagina12.com.ar/diario/elmundo/4-67637-2006-05-31.html, http://www.clarin. com/diario/2006/05/31/elmundo/i-02001.htm.

Ley organica Constitucional, s.o. C I.

62

Vgl. Art. 19 Nr. 10 der Politischen Verfassung der Republik Chile.

Zur Situation des chilenischen Bildungssystems und seiner historischen Entwicklung aus der Perspektive der "Critical Educational Studies" vgl. Jill Pinkney Pastrana, Subtle Tortures of the Neo-liberal Age: Teachers, Students, and the Political Economy of Schooling in Chile, Journal for Critical Education Policy Studies 5 N. 2 (11/2007).

64 http://www.consejoeducacion.cl/view/viewArticulos.asp?idArticulo=84.

65 Vgl. etwa http://lanacion2007.altavoz.net/prontus_noticias_v2/site/artic/20090304/pags/ 20090304011621.html, http://www.senado.cl/prontus_galeria_noticias/site/artic/20081209/pags/ 20081209211846.html.

66

http://www.leychile.cl/Navegar?idNorma=1006043\&idParte=\&idVersion=2009-09-12. Siehe auch: http://www.bcn.cl/guias/ley-general-educacion. 
che die Qualität der in weiten Teilen privatwirtschaftlich organisierten Bildungseinrichtungen gewährleisten sollen. Den Ansprüchen der Reformbefürworter genügen die Neuerungen allerdings nicht - der Widerstand gegen das aus Zeiten der Diktatur stammende LOCE scheint sich nun in nahezu gleicher Form und Intensität gegen das LGE zu richten: ${ }^{67}$ Dessen Kritiker wenden sich insbesondere gegen die weiterhin vorherrschend marktwirtschaftliche Ausrichtung des Bildungssystems und die unverändert subsidiäre Rolle des Staates, wodurch die gesellschaftliche Ungleichverteilung von Bildungschancen weiter vertieft werde. $^{68}$ Mit der Begründung einer auch durch das LGE weiterhin unzureichenden Gewährleistung des Rechts auf Zugang zu Bildung werden auch verfassungsrechtliche Bedenken gegen das Gesetz vorgebracht.

\section{Reform des Grundrechtskatalogs}

Auch die Kritik am Grundrechtssystem der geltenden Verfassung entzündet sich vorwiegend an dessen neoliberaler Ausrichtung, ${ }^{70}$ die sich in der hauptsächlich abwehrrechtlichen Konzeption von Grundrechten und der einseitigen Gewichtung des Schutzes ökonomischer Interessen - insbesondere des Eigentums - durch Verfassung und vorherrschende Dogmatik äußere. ${ }^{71}$ Soziale und ökonomische Grundrechte im Sinne von Leistungs- oder Teilhaberechten würden im geltenden Verfassungsrecht dagegen - wenn überhaupt - nur nachrangig berücksichtigt. ${ }^{72}$ Der Grundrechtskatalog, der im Gegensatz zum Organisationsteil der Verfassung auch im Rahmen der umfassenden Reform im Jahr 2005 nicht maßgeblich verändert wurde, spiegle so weiterhin die Prägung der ursprünglichen Verfassung als normativem Ausdruck des durch die marktliberalen Ökonomen des Militärregimes definierten Wirtschaftsmodells wider, welches sich am neoliberalen Programm der Chicagoschule orientiert habe. ${ }^{73}$ Um die rechtliche Anerkennung der ökonomischen, sozialen und kulturellen Grundrechte sowie der „Menschenrechte der dritten Generation“ zu gewährleisten, werden, neben der Möglichkeit einer dogmatischen Weiterentwicklung und Neuinterpretation des bestehenden Grundrechtskatalogs im Sinne einer „,demokratischen und liberalen“

67

68

69

70

71

72

73

Siehe http://ipsnoticias.net/nota.asp?idnews=91726.

$\mathrm{Zu}$ den Argumenten der Gegner des LGE siehe: http://www.caletasur.cl/BOLETIN/Declaraci\% F3n\%20LGE\%202009.pdf, http://www.opech.cl/editoriales/2009_08/index_17_08_09_nota_ promulgacion_lge.pdf.

http://www.radio.uchile.cl/notas2.asp?idNota=54565.

Vgl. Juan Carlos Ferrada Bórquez, La Constitución Económica de 1980. Algunas Reflexiones Críticas. Revista de Derecho (Valdivia) 11 (2000), S. 50.

Vgl. Ruiz Tagle-Vial, Fn. 34; ders., Una visión democratica y liberal de los derechos fundamentals para la Constitución chilena del Bicentenario, in: Andrés Bordalí Salamanca (Hrsg.) Justicia Constitucional y Derechos Fundamentales, Santiago de Chile 2006, S. 120.

Zúñiga Urbina, Fn. 49, S. 357.

Vgl. Ferrada Borquez, Fn. 70, S. 51, 53; Cea, in: Simposio: Reformas del ano 2005 a la Constitución, Fn. 5, 77, Zúñiga Urbina, Fn. 49, S. 351. 
Grundrechtskonzeption unter Heranziehung etwa auch der internationalen Menschenrechtskataloge, ${ }^{74}$ Verfassungsreformen „,der zweiten Generation““75 vorgeschlagen.

\section{Geltung des Völkerrechts}

In einer Entscheidung aus dem Jahr 2002 erklärte der chilenische Verfassungsgerichtshof das Romstatut des Internationalen Strafgerichtshofs nur kurz vor seinem Inkrafttreten für verfassungswidrig. ${ }^{76}$ Die Mehrheit der Verfassungsrichter war der Überzeugung, dass das Statut, indem es dem zu begründenden Gerichtshof als einem supranationalen Organ die Kompetenz zur Ausübung der Rechtsprechung verleihe, in verfassungsmodifizierender Form in Kompetenzen und Funktionen der Judikative eingreife. Da die erforderliche Transformation des Statuts in das innerstaatliche Recht so in einer durch die Verfassung nicht vorgesehenen Weise Souveränitätsrechte transferiere, habe dieser eine Änderung der entsprechenden Verfassungsnormen vorauszugehen. ${ }^{77}$ Nach mehr als sieben Jahre währenden Reformbestrebungen und einer ausgeprägten politischen Debatte ${ }^{78}$ konnte die erforderliche Verfassungsänderung Ende Mai 2009 verabschiedet werden. ${ }^{79}$ Chile hat den Vertrag von Rom am 17.6.2009 ratifiziert.

Das Urteil ist, ebenso wie der langwierige Ratifikationsprozess, Konsequenz der nach Auffassung des Verfassungsgerichts ${ }^{80}$ und eines Teils der Lehre im chilenischen Verfassungsrecht angelegten konsequent dualistischen, das Primat der Verfassung statuierenden, Konzeption des Verhältnisses von Völkerrecht und nationalem Recht. ${ }^{81}$ Internationale Verträge bedürfen demnach zur Begründung ihrer innerstaatlichen Geltung der Inkorporierung in das nationale Recht nach den für die entsprechende Materie vorgesehenen Verfahren, nehmen in der Gesetzeshierarchie den Rang des Transformationsgesetzes an und stehen damit generell unter der Verfassung; ins nationale Recht überführte internationale

Ruiz Tagle-Vial, una visión democratica y liberal de los derechos fundamentales, Fn. 71, insbes.

S. $110 \mathrm{ff} ., 126 \mathrm{ff}$.

75

76

77

78

79

Urteil vom 8.4.2002, rol número 346.

Vgl. Humberto Nogueira Alcalá, Consideraciones sobre el fallo del Tribunal Constitucional respecto del Tratado de Roma que establece la Corte Penal Internacional, Anuario de Derecho Constitucional Latinoamericano 2002, S. 450.

Vgl. http://www.tribunalpenalinternacional.cl/documentos/ratificacion_er_cpi.pdf, http://www. tribunalpenalinternacional.cl/ver_post.php?i=31, http://adhb.wordpress.com/2009/03/10/es-unaverguenza-que-chile-no-sea-capaz-de-ratificar-el-cpi/.

Eine Dokumentation des Gesetzgebungsverfahrens ist unter http://www.bcn.cl/histley/lfs/hdl20352/HL20352.pdf abrufbar.

80 Vgl. Nogueira Alcalá, Fn. 77, S. 451 f.

81

Teodoro Ribera Neumann, Los Tratados Internacionales y su control a posteriori por el Tribunal Constitucional, Estudios Constitucionales (Universidad de Talca) 2007, S. 115 f. 
Verträge bedürfen der verfassungskonformen Auslegung. ${ }^{82}$ Ein solches souveränitätszentriertes Verständnis des Verhältnisses von staatlichem Recht und Völkerrecht ist allerdings auch in Chile alles andere als unbestritten: Die Rechtssprechung des obersten Gerichtshofes ${ }^{83}$ anerkennt insbesondere die völkerrechtsvertraglich verbürgten Menschenrechte als generelle Beschränkungen der staatlichen Souveränität Chiles, deren innerstaatliche Geltung auch verfassungsändernden Mehrheiten nicht zugänglich sei, ${ }^{84}$ ebenso die völkerrechtsdogmatischen Konzepte des ius cogens und der Verpflichtungen erga omnes. ${ }^{85}$ Auch eine Reihe von Autoren interpretieren die - vor allem im Zuge der Reform des Jahres 2005 hinzugekommenen - Regelungen der Verfassung in diesem Sinne und ziehen entsprechende Schlüsse, etwa hinsichtlich der übergeordneten Stellung internationaler Verträge in der Gesetzeshierarchie, der unmittelbaren Wirkung völkerrechtlicher Menschenrechtsverbürgungen oder der fehlenden Kompetenz des Verfassungsgerichts zur Kontrolle internationaler Vertragswerke a posteriori. ${ }^{86}$

Die gegensätzlichen Auffassungen über die innerstaatliche Geltung völkerrechtlicher Verpflichtungen zeigen aktuell in der Diskussion um die Rechte der indigenen Bevölkerung praktische Auswirkungen: Nachdem Chile die Konvention 169 der Internationalen Arbeitsorganisation betreffend die Rechte der Indigenen Völker im Jahr 2008 unterzeichnet hat und diese im September 2009 in Kraft getreten ist, ${ }^{87}$ entspinnt sich nunmehr eine kontroverse Debatte um die verfassungsrechtliche Umsetzung der dort verbürgten Rechte. Intensive Kritik entzündet sich insbesondere an dem Vorschlag der zuständigen Senatskommission zu den in diesem Zusammenhang anstehenden Verfassungsänderungen, ${ }^{88}$ der insbesondere konventionswidrig die Anerkennung der indigenen „Völker“ als Rechtssubjekte verweigere ${ }^{89}$ und deren ebenfalls durch die Konvention verbürgtes Recht auf Konsul-

Ribera Neumann, Fn. 81, S. 97 f., 115 f.

Corte Suprema de Justicia.

Zur divergierenden Rechtsprechung von Verfassungsgericht und Oberstem Gerichtshof siehe Humberto Nogueira Alcalá, Reforma Constitucional de 2005 y control de constitucionalidad de Tratados Internacionales, Estudios Internacionales 5 (2007), S. 73 ff.

Gonzalo Aguilar Cavallo, La Corte Suprema y la aplicación del Derecho Internacional: un proceso esperanzador, Estudios Constitucionales 2009, S. 94, 127.

Siehe Nogueira Alcalá, Fn. 84, S. 86; Ana María García Barzelatto, Informe en Derecho. Control de Constitucionalidad de los Tratados Internacionales con especial referencia al control represivo, Estudios Constituciónales 5 (2007), S. 473 ff.

Siehe http://www.oitchile.cl/noticias.php?id=196, http://www.lanacion.cl/entro-en-vigencia-elconvenio-169-de-la-oit/noticias/2009-09-16/003055.html.

Vgl. das entsprechende Gutachten der Kommission abrufbar unter http://www.politicaspublicas. net/panel/reforma/documentos/213-informe-sobre-proyectos-de-reforma-constitucional-relativa-apueblos-indigenas.html. Eine umfangreiche Dokumentation der Reformvorschläge ist unter http://www.politicaspublicas.net/panel/reforma/documentos/386-indicaciones.html zu finden.

Als Rechtssubjekte anerkannt werden stattessen die Rechte „der indigenen Gemeinschaften, Organisationen und ihrer Mitglieder“. Siehe hierzu Bartolomé Clavero, Chile. Reforma con- 
tation im Rahmen der anstehenden Reformen missachte. ${ }^{90}$ Das Senatsgutachten belegt tatsächlich eine sehr restriktive Interpretation der Geltung der Konventionsverbürgungen, indem es etwa betont, dass deren „Regelungen der nationalen Gesetzgebung und den Verbürgungen der Verfassung nicht zuwiderlaufen können“ und die Abhängigkeit der einzelnen völkerrechtlich gewährleisteten Rechte von ihrer Übereinstimmung mit der Verfassung und den bestehenden einfachgesetzlichen Regelungen immer wieder hervorhebt. ${ }^{91}$ Die geplanten Verfassungsänderungen bleiben nach der Auffassung ihrer Kritiker daher weit unter den internationalen Standards ${ }^{92}$ und stoßen auf die kategorische Ablehnung indigener Organisationen. ${ }^{93}$ Um die problematische Frage der Geltung insbesondere der völkerrechtlichen Menschenrechtsverbürgungen ${ }^{94}$ zu klären und eine einheitliche, völkerrechtskonforme Rechtsprechung zu ermöglichen, ${ }^{95}$ schlagen Reformbefürworter etwa die Inkorporierung einer Regelung zum Vorrang des Völkerrechts vor. ${ }^{96}$

\section{Eine gänzlich neue Verfassung für Chile?}

In der Folge der großen Verfassungsreform des Jahres 2005 entzündete sich in der chilenischen Rechtswissenschaft und Politik die Diskussion, ob es sich bei dem zum damaligen Zeitpunkt immerhin achtzehnfach reformierten Regelwerk nunmehr um eine ,neue“ Verfassung handle, oder diese vielmehr weiterhin, trotz aller Reformen, dem weitgehenden Ende der ,,autoritären Enklaven“ und einer in großen Teilen erneuerten Institutionenordnung, die ,alte“, unter Pinochet erarbeitete und implementierte Politische Verfassung von

stitucional cancelatoria de Derechos Indígenas, http://clavero.derechosindigenas.org/?p=1154. Der Reformvorschlag betont an erster Stelle die „Einheit und Unteilbarkeit“ der „,multikulturellen Nation“, s.o. Fn. 88.

Vgl. Art. 6 Nr. 1 a) der Konvention. S.a. http://www.politicaspublicas.net/panel/reforma/ declaraciones/273-reforma-Constituciónal-comunidades-mapuche-de-lanco-rechazan-proceso-deconsulta-viciado.html.

Siehe das Gutachten der Senatskommission, s.o. Fn. 88, S. 7, 13, 15; S.a. Clavero, Fn. 89.

Vgl. http://www.elciudadano.cl/2009/03/10/abogado-jose-aylwin-\%E2\%80\%9CreconocimientoConstituciónal-de-los-pueblos-indigenas-es-enganoso-y-esta-muy-por-debajo-de-los-estandaresinternacionales $\% \mathrm{E} 2 \% 80 \% 9 \mathrm{D} /$.

http://aureliennewenmapuche.blogspot.com/2009/03/organizaciones-indigenas.html, http://www. minkandina.org/index.php?news $=41$

Vgl. den Bericht des UN Human Rights Council, Working Group on the Universal Periodic Review, Fifth Session, Geneva 15.4. 2009, abrufbar unter: http://www.scribd.com/doc/14034073/ EPU-CHILE-2009-Observaciones-ONGs, S. 2.

Siehe, bezogen auf fehlende Regelungen zur gerichtlichen Umsetzung der Rechtsprechung des Interamerikanischen Gerichthofes, Humberto Nogueira Alcalá, Los desafíos de la sentencia de la 96 Corte Interamericana en el caso Almonacid Arellano, Ius et Praxis 12 (2006), S. 382.

Zúñiga Urbina, Fn. 49, S. 363. S.a. Nogueira Alcalá, Fn. 77, S. 466. 
1980 darstelle. $^{97}$ Bedeutsam ist diese Debatte insbesondere im Zusammenhang mit der Frage nach der politischen Legitimität der geltenden Verfassung, ${ }^{98}$ die von den Befürwortern eines neu ins Leben zu rufenden Verfassungsprojekts generell verneint wird. Die bisherige schrittweise Verfassungsreform zur Umsetzung des weithin anerkannten Änderungsbedarfs, wie sie seit dem Ende der Militärdiktatur praktiziert und weiterhin als angemessene Vorgehensweise zur politischen Modernisierung Chiles vorgeschlagen und praktiziert wird, war nach deren Auffassung nicht in der Lage, den autoritären Kern der Verfassung zu ändern; diese sei vielmehr im gleichen Maß in ihrer Substanz unverändert geblieben, wie sie reformiert worden sei. ${ }^{99}$ Die Verfassung erschwere nicht nur substanzielle Reformen der seit Pinochets Regime fortbestehenden politischen, wirtschaftlichen und gesellschaftlichen Ordnung, indem diese mit hohen Anforderungen an parlamentarische Mehrheiten verbinde. ${ }^{100}$ Das Projekt sukzessiver Verfassungsreformen sei vielmehr prinzipiell zum Scheitern verurteilt, weil so das „Rückgrat“, die bereits in ihren Grundstrukturen angelegte antidemokratische und neoliberale Ausrichtung der Verfassung der Militärjunta, nicht verändert werden könne. ${ }^{101}$

Um Chiles Übergang zur Demokratie zu vollenden und den eigentlichen Geburtsfehler der Politischen Verfassung, ${ }^{102}$ ihren illegitimen Ursprung, zu beseitigen, bedarf es aus dieser Sicht eines gänzlich neuen Verfassungsprojektes, das mittels geeigneter, die verfassungsgebende Gewalt des Volkes möglichst unmittelbar verwirklichender Verfahren demokratische Legitimität herzustellen habe. ${ }^{103}$ Anstelle weiterer „kosmetischer Veränderungen“ am alten Verfassungstext, ${ }^{104}$ fordern dementsprechend eine Vielzahl zivilgesellschaftlicher Vereinigungen, Politiker und Juristen die Erarbeitung einer neuen Verfassung im parla-

97

Teilweise wird diese Frage so beantwortet, dass die Verfassung nunmehr in ihrem Organisationsteil eine neue „Philosophie“ widerspiegle, wohingegen insbesondere der Grundrechtskatalog weiterhin der Ideologie des Militärregimes entspreche, vgl. Zúñiga Urbina, Fn. 49, S. 350 f., 365, s.a.o. C. VI.

98

99

100

Zúñiga Urbina, Fn. 49, S. 359 f.

Vgl. Pablo Ruiz-Tagle, La trampa del neopresidencialismo: la constitucion gatopardo, in: Renato Cristi/Pablo Ruiz Tagle, La Republica en Chile, teoría y práctica del constitucionalismo republicano, Santiago de Chile 2006, S. 197 ff., der die Verfassung, in Anspielung auf den Roman „Der Leopard“ (,El Gatopardo“) von G. T. di Lampedusa, aufgrund ihrer Eigenschaft, trotz aller demokratischer Reformen ihren autokratischen Charakter in aller Schärfe zu bewahren, als „Constitución gatopardo" bezeichnet.

S.o. C I.

101 Vgl. Graciela Alvarez Rojas, Beitrag zum Seminar „Nececita Chile una nueva Constitución?“”v. 25.8.2008, Universidad Central, Santiago de Chile stattfand, abrufbar unter http://www. observatoriogeneroyliderazgo.cl/index.php?option=com_content\&task=view\&id=566\&Itemid=2; Jorge Tapia, in: Anuario de Derechos Humanos 2006, Fn. 5, S. 82; Ruiz-Tagle, Fn. 99.

103 Vgl. Zúñiga Urbina, Fn. 49, S. 359 f.

104 Vgl. Tapia, Fn. 101, S. 81. 
mentarischen Kontext oder die Abhaltung einer verfassungsgebenden Versammlung. ${ }^{105}$ Inwiefern solche, oder ähnlich konsequente Reformprojekte nach den 20 Jahre währenden Reformbemühungen der Concertación nun unter dem neuen Präsidenten Piñera - der aus dem Lager derjenigen rechten Parteien stammt, die als politische Profiteure der Verfassung von 1980 während Chiles langwierigem Übergang zur Demokratie grundlegende Reformen stets zu verhindern oder zumindest abzumildern wussten - realisierbar sein werden, bleibt abzuwarten. ${ }^{106}$ Vorläufig jedenfalls wird Pinochets nunmehr siebenundzwanzigfach reformierte Verfassung auch weiterhin die institutionellen Spielregeln vorgeben, welche die politischen Möglichkeiten - auch ihrer eigenen - Reform entscheidend bedingen und beschränken.

Vgl. etwa den Reformvorschlag des unterlegenen Präsidentschaftskandidaten der Concertación, Eduardo Frei Ruiz Tagle, http://www.archivochile.com/Chile_actual/elecciones_2009/frei/doc_ part/frei_part0032.pdf, http://www.movimientoporlaconstituyente.cl/; http://www. asambleaconstituyentechile.cl/noticias/constitucion-chile-entre-el-cambio-y-la-reforma, http:// 106 asambleaconstituyente.cl/, http://asambleachile.tk/, http://chilenosconstituyente.blogspot.com/.

\footnotetext{
Vgl. http://www.lanacion.cl/pinera-al-pizarron-por-reformas-politicas/noticias/2010-02-16/
} 221448.html. 